\title{
Albumin-Bilirubin (ALBI) Grade as Part of the Evidence-Based Clinical Practice Guideline for HCC of the Japan Society of Hepatology: A Comparison with the Liver Damage and Child-Pugh Classifications
}

\author{
Atsushi Hiraoka ${ }^{a}$ Takashi Kumada $^{b}$ Masatoshi Kudo ${ }^{c}$ Masashi Hirooka $^{d}$ \\ Kunihiko Tsuji ${ }^{\mathrm{e}}$ Ei Itobayashi ${ }^{f}$ Kazuya Kariyamag ${ }^{9}$ Toru Ishikawah \\ Kazuto Tajiri ${ }^{i}$ Hironori Ochi ${ }^{j}$ Toshifumi Tada ${ }^{b}$ Hidenori Toyodab \\ Kazuhiro Nouso ${ }^{g}$ Kouji Joko ${ }^{j}$ Hideki Kawasaki ${ }^{a}$ Yoichi Hiasad \\ Kojiro Michitaka ${ }^{a}$ on behalf of the Real-Life Practice Experts for HCC (RELPEC) \\ Study Group and HCC 48 Group (hepatocellular carcinoma experts from 48 clinics) \\ ${ }^{a}$ Gastroenterology Center, Ehime Prefectural Central Hospital, Ehime, ${ }^{b}$ Department \\ of Gastroenterology and Hepatology, Ogaki Municipal Hospital, Gifu, 'Department of \\ Gastroenterology and Hepatology, Kinki University Faculty of Medicine, Osaka-Sayama, \\ ${ }^{\mathrm{d}}$ Department of Gastroenterology and Metabology, Ehime University Graduate School of \\ Medicine, Ehime, ${ }^{e}$ Center of Gastroenterology, Teine Keijinkai Hospital, Sapporo, ${ }^{f}$ Department of \\ Gastroenterology, Asahi General Hospital, Asahi, g Department of Gastroenterology, Okayama City \\ Hospital, Okayama, h' Department of Gastroenterology, Saiseikai Niigata Daini Hospital, Niigata, \\ 'Department of Gastroenterology, Toyama University Hospital, Toyama, and \\ jHepato-Biliary Center, Matsuyama Red Cross Hospital, Matsuyama, Japan
}

\section{Keywords}

ALBI grade $\cdot$ Indocyanine green $\cdot$ Liver damage $\cdot$ Child-Pugh $\cdot$ Hepatocellular carcinoma

\begin{abstract}
Aim/Background: The purpose of this study was to evaluate the validity of 3 classifications for assessing liver function, the liver damage and Child-Pugh classifications and the newly proposed albumin-bilirubin (ALBI) grade, in order to examine the feasibility of evaluating hepatic function using ALBI grade with the hepatocellular carcinoma (HCC) treatment algorithm used in Japan. Methods: We analyzed the medical records of 3,495 Japanese HCC patients
\end{abstract}

See related article by Kudo: Albumin-Bilirubin Grade and Hepatocellular Carcinoma Treatment Algorithm. DOI 10.1159/000462199, published on pp. 185-188. 


\section{Liver Cancer}

\begin{tabular}{l|l}
\hline Liver Cancer 2017;6:204-215 \\
\hline DOI: 10.1159/000452846 & $\begin{array}{l}\text { @ 2017 S. Karger AG, Basel } \\
\text { www.karger.com/lic }\end{array}$ \\
\hline
\end{tabular}

admitted from 2000 to 2015, which were comprised of 1,580 patients hospitalized in the Ehime Prefecture area and used as a training cohort (Ehime group), and 1,915 others who were used for validation (validation group). ALBI score used for grading ( $\leq-2.60=$ grade 1 , greater than -2.60 to $\leq-1.39=$ grade 2 , greater than $-1.39=$ grade 3 ) as well as clinical features and prognosis (Japan Integrated Staging [JIS], modified JIS, ALBI-TNM [ALBI-T] score) were retrospectively investigated. Results: For prediction of liver damage $A$, the values for sensitivity and specificity, positive predictive and negative predictive values, and positive and negative likelihood ratios of ALBI-1 and Child-Pugh A were similar among the 2 groups. Akaike information criterion results showed that prognosis based on ALBI grade/ALBI-T score was better than that based on liver damage/modified JIS score and Child-Pugh/JIS score (22,291.8/21,989.4, $22,379.6 / 22,076.0,22,392.1 / 22,075.1$, respectively). The cutoff values for ALBI score for indocyanine green retention rate at $15 \mathrm{~min}$ (ICG-R15) $<10,<20$, and $<30 \%$ were -2.623 (area under the curve $[A \cup C]: 0.798),-2.470$ (AUC: 0.791 ), and -2.222 (AUC: 0.843 ), respectively. The distribution of ICG-R15 ( $<10 \%, 10$ to $<20 \%, 20$ to $<30 \%$, and $\geq 30 \%$ ) for ALBI grade 1 was similar to that for liver damage $A$. There were only small differences with regard to therapeutic selection with the Japanese HCC treatment algorithm between liver damage and ALBI grade. Conclusion: ALBI grade is a useful and easy classification system for assessment of hepatic function for therapeutic decision making.

(C) 2017 S. Karger AG, Basel

\section{Introduction}

In Japan, the degree of liver damage as a guide to liver function (liver damage classification) [1] has been defined based on ascites, serum bilirubin, serum albumin, indocyanine green retention rate at 15 min (ICG-R15) (\%), and prothrombin time activity (\%) by the Liver Cancer Study Group of Japan (LCSGJ) (Table 1). The liver damage classification is utilized for evaluation of hepatic function with the hepatocellular carcinoma (HCC) treatment algorithm of the evidence-based Japan Society of Hepatology (JSH) guideline [2, 3]. Moreover, ICG-R15 has been reported to be a useful marker for predicting the hepatic function of a safely resectable segment in patients with HCC [4-6]. However, ICG-R15 data are not easily obtained in all patients, because ICG injection is needed. On the other hand, the Child-Pugh classification [7] is used worldwide for evaluation of hepatic function in patients with liver cirrhosis. As for the weak points of the Child-Pugh classification, some factors are subjective (ascites, encephalopathy), while interrelated factors (serum albumin, ascites) also exist. Omagari et al. [8] reported that the liver damage classification is a more effective evaluation method for patients with early stage HCC as compared to the Child-Pugh classification. Recently, a simple evaluation method for hepatic function, termed albumin-bilirubin (ALBI) grade, which is calculated using only serum albumin and total bilirubin, has been proposed [9], and some have reported its usefulness for HCC treatment planning [9-16].

ALBI grade [13] and liver damage [8] have been reported to have better capability for evaluation of hepatic function in Japanese HCC patients as compared to the Child-Pugh classification, though few studies have assessed the correlation between them. In the present study, we investigated the clinical usefulness of the ALBI grade by comparing it with both the liver damage and Child-Pugh classifications to analyze whether the ALBI grade can be substituted for the liver damage classification in the evidence-based JSH guideline. 


\section{Liver Cancer}

Table 1. Degree of liver damage used by LCSGJ

\begin{tabular}{l|l}
\hline Liver Cancer 2017;6:204-215 \\
\hline DOI: 10.1159/000452846 & $\begin{array}{l}\text { @ 2017 S. Karger AG, Basel } \\
\text { www.karger.com/lic }\end{array}$ \\
\hline
\end{tabular}

Hiraoka et al.: ALBI Grade for HCC Treatment

\begin{tabular}{llll}
\hline & LD A & LD B & LD C \\
\hline Ascites & none & controllable & uncontrollable \\
Serum bilirubin, mg/dL & $<2.0$ & $2.0-3.0$ & $>3.0$ \\
Serum albumin, g/dL & $>3.5$ & $3.0-3.5$ & $<3.0$ \\
ICG-R15, \% & $<15$ & $15-40$ & $>40$ \\
Prothrombin activity, \% & $>80$ & $50-80$ & $<50$ \\
\hline
\end{tabular}

The degree of liver damage is decided based on the highest grade that contains at least 2 findings. When there are factors $B$ and $C$, the degree is recommended as class B. LD, liver damage; ICG-R15, indocyanine green retention rate after $15 \mathrm{~min}$.

\section{Materials and Methods}

\section{Patients}

From 2000 to 2015, 3,495 patients with naive HCC were examined using ICG-R15 at 9 different institutions (Ehime Prefectural Hospital [ $n=940]$, Ehime University Hospital [ $n=268]$, Matsuyama Red Cross Hospital [ $n=372]$, Ogaki Municipal Hospital [ $n=844]$, Okayama City Hospital [ $n=135]$, Teine Keijinkai Hospital [ $n=546]$, Asahi General Hospital [ $n=124]$, Saiseikai Niigata Hospital [ $n=111]$, Toyama University Hospital $[n=155])$. After receiving informed consent, ICG-R15 was determined following a bolus injection of ICG $(0.5 \mathrm{mg} / \mathrm{kg})$ prior to therapy for HCC. ALBI score was calculated based on serum albumin and total bilirubin using the following formula, ALBI-score: $\left(\log _{10}\right.$ bilirubin $\left.[\mu \mathrm{mol} / \mathrm{L}] \times 0.66\right)+($ albumin $[\mathrm{g} / \mathrm{L}] \times-0.085)$, while ALBI grade was defined by the resulting score $(\leq-2.60=$ grade 1 , greater than -2.60 to $\leq-1.39=$ grade 2, greater than -1.39 = grade 3) [9].

Patients positive for anti-hepatitis $\mathrm{C}$ virus (HCV) were judged to have $\mathrm{HCC}$ due to $\mathrm{HCV}$, and those positive for hepatitis B virus surface antigen (HBsAg) were judged to have HCC due to hepatitis B virus (HBV). Those without anti-HCV and HBsAg were defined as negative for both HBV and HCV (nonBnonC).

\section{Comparison between Three Methods for Evaluation of Hepatic Function}

We examined the relationship between the liver damage (Table 1) and Child-Pugh classifications and the ALBI grade using a validation cohort. Patients treated at institutions located in Ehime Prefecture (Ehime Prefectural Hospital, Ehime University Hospital, Matsuyama Red Cross Hospital) were defined as the training cohort ( $n=1,580$, Ehime group), while the others were used as the validation cohort ( $n=1,915$, validation group). For predicting liver damage class A, which often has good values of ICG-R15, the sensitivity, specificity, positive predictive value (PPV), negative predictive value (NPV), positive likelihood ratio, and negative likelihood ratio of ALBI grade 1 and Child-Pugh class A were estimated in both groups.

The number of patients was investigated in each arm of the therapeutic option of the evidence-based JSH guideline [2, 3], with each grade used for assessment of hepatic function. In addition, we retrospectively evaluated the prognosis shown by each classification for hepatic function, and the prognosis shown by 3 different prognostic staging systems [Japan Integrated Staging (JIS) score, modified JIS score, ALBI-TNM (ALBI-T) score]. The distribution of ICG-R15 in each classification of hepatic function was investigated. JIS score was calculated using TNM stage of LCSGJ and Child-Pugh class $[17,18]$, modified JIS score using TNM stage of LCSGJ and liver damage [19], and ALBI-T using TNM stage of LCSGJ and ALBI grade [13].

Diagnosis and Surveillance of HCC Patients

HCC was diagnosed based on an increasing course of $\alpha$-fetoprotein, as well as dynamic CT [20], magnetic resonance imaging, contrast-enhanced US with perflubutane (Sonazoid ${ }^{\circledR}$, Daiichi Sankyo Co., Ltd., Tokyo, Japan) [21], and/or pathological findings. TNM stage was determined as reported in studies for staging of HCC conducted by the LCSGJ, ed 6 [1].

\section{Treatment Criteria}

After 2005, all treatments were performed following the Japanese practical guidelines for HCC [2, 3], whenever possible. All treatments were performed after obtaining written informed consent from the 
Hiraoka et al.: ALBI Grade for HCC Treatment

patient. The study protocol was approved by the Institutional Ethics Committee of Ehime Prefectural Central Hospital (No. 28-30).

\section{Statistical Analysis}

Data are expressed as median values or interquartile range (IQR). Statistical analyses were performed using the Student $t$ test, Mann-Whitney U test, $\chi^{2}$ test, Pearson test, log-rank test, and Kaplan-Meier methods. A $p$ value $<0.05$ was considered to indicate statistical significance. Akaike information criterion (AIC) for prognosis with each method, receiver operating characteristic (ROC), and the area under the curve (AUC) were calculated for comparisons between the ALBI score and ICG-R15. All statistical analyses were performed using Easy R (EZR) (Saitama Medical Center, Jichi Medical University, Saitama, Japan) [22], a graphical user interface for R (The R Foundation for Statistical Computing, Vienna, Austria) [23].

\section{Results}

\section{Clinical Backgrounds of Enrolled Patients}

The total number of patients diagnosed with and/or treated for HCC from 2000 to 2015 at our institutions was 7,565, of whom 3,495 (46.2\%; 2,488 males, 1,007 females; median age 70 years old, IQR 62-75 years) were enrolled in the present study. Their clinical backgrounds are shown in Table 2. HCV was the diagnosis in 2,257, HBV in 506, HBV and HCV in 18, and nonBnonC in 714 patients. In the comparisons between the Ehime and validation groups, there were significant differences with regard to gender, AST, ALT, platelets, total bilirubin, ascites, encephalopathy, ICG-R15, liver damage classification, Child-Pugh classification, ALBI grade, tumor number (single and multiple), $\alpha$-fetoprotein $(\geq 100 \mathrm{ng} / \mathrm{mL}$ ), des- $\gamma$-carboxy prothrombin, and therapies (Table 2).

Surgical resection was performed in $1,274(36.5 \%)$ patients, radiofrequency ablation (RFA) [24, 25] in 1,652 (47.3\%), percutaneous ethanol injection in $51(1.5 \%)$, and transcatheter arterial chemoembolization in 462 (13.2\%), while other treatments including supportive care were performed in $56(1.6 \%)$.

Grade distribution for each of the 3 hepatic function assessment methods is shown in Table 3. In the Ehime group, the values for sensitivity, specificity, PPV, NPV, positive likelihood ratio, and negative likelihood ratio for predicting liver damage class A based on ALBI grade 1 were $0.677,0.851,0.831,0.708,4.534$ (95\% CI: 3.800-5.409), and 0.380 (95\% CI: 0.343-0421), respectively, while those based on Child-Pugh class A were 0.999, $0.413,0.649,0.997,1.703$ (95\% CI: 1.604-1.808), and 0.003 (95\% CI: 0.000-0.021), respectively. In the validation group, those values for predicting liver damage class A based on ALBI grade 1 were $0.632,0.906,0.908,0.628,6.739$ (95\% CI: 5.393-8.422), and 0.406 (95\% CI: $0.375-0.439$ ), respectively, and those based on Child-Pugh class A were 0.995, $0.438,0.721,0.983,0.769,1.771$ (95\% CI: 1.664-1.885), and 0.012 (95\% CI: 0.005-0.027), respectively. Similar tendencies for these values were seen in both groups. For all 3,495 enrolled patients, the values for sensitivity, specificity, PPV, NPV, positive likelihood ratio, and negative likelihood ratio for predicting liver damage class A based on ALBI grade 1 were $0.651,0.879,0.873,0.664,0.751,5.373$ (95\% CI: 4.677-6.171), and 0.397 (95\% CI: $0.373-0.423$ ), respectively, and those based on Child-Pugh class A were 0.996, 0.426, $0.689,0.989,0.746,1.736$ (95\% CI: 1.663-1.813), and 0.008 (95\% CI: 0.004-0.018), respectively.

When each grade was used for assessment of hepatic function with the evidence-based JSH guideline, after exclusion of patients with major vein invasion, extrahepatic metastasis, and/or rupture $(n=209)$, the numbers of patients in arms $1,2,3,4$, and 5 of the algorithm with the use of the liver damage classification were 2,138, 554, 257, 203, and 134, respectively, while the numbers of patients in those arms using the Child-Pugh classification were 


\section{Liver Cancer}

\begin{tabular}{l|l}
\hline Liver Cancer 2017;6:204-215 \\
\hline DOI: 10.1159/000452846 & $\begin{array}{l}\text { @ 2017 S. Karger AG, Basel } \\
\text { www.karger.com/lic }\end{array}$ \\
\hline
\end{tabular}

Hiraoka et al.: ALBI Grade for HCC Treatment

Table 2. Clinical backgrounds of patients and comparisons between the Ehime and validation groups

\begin{tabular}{|c|c|c|c|}
\hline & $\begin{array}{l}\text { Ehime group } \\
(n=1,580)\end{array}$ & $\begin{array}{l}\text { Validation group } \\
(n=1,915)\end{array}$ & $\begin{array}{l}\text { Total } \\
(n=3,495)\end{array}$ \\
\hline Age, years ${ }^{a}$ & $69(62-76)$ & $70(63-75)$ & $70(62-75)$ \\
\hline Gender, male:female & $1,111: 469$ & $1,377: 538^{* *}$ & $2,488: 1,007$ \\
\hline Etiology, HCV:HBV:HBV\&HCV:nonBnon C & $1,107: 166: 12: 295$ & $1,150: 340: 6: 419$ & $2,257: 506: 18: 714$ \\
\hline AST, IU/L ${ }^{a}$ & $52(36-76)$ & $47(33-67)^{* *}$ & $50(35-72)$ \\
\hline ALT, IU/L ${ }^{a}$ & $42(27-66)$ & $40(26-63)^{*}$ & $41(27-64)$ \\
\hline Platelets, $\times 10^{4}$ cells $/ \mu \mathrm{L}^{\mathrm{a}}$ & $11.0(7.8-15.4)$ & $11.9(8.4-16.4)^{* *}$ & $11.4(8.1-15.8)$ \\
\hline Total bilirubin, $\mathrm{mg} / \mathrm{dL}^{\mathrm{a}}$ & $0.8(0.6-1.1)$ & $0.7(0.6-1.0)^{* *}$ & $0.8(0.6-1.0)$ \\
\hline Albumin, $\mathrm{g} / \mathrm{dL}^{\mathrm{a}}$ & $3.8(3.4-4.2)$ & $3.8(3.5-4.1)$ & $3.8(3.4-4.1)$ \\
\hline Prothrombin time, $\%^{\mathrm{a}}$ & $84.1(73.2-95.5)$ & $87.6(75.0-96.7)$ & $86.0(74.0-96.0)$ \\
\hline Ascites, none:mild:moderate to severe & $1,442: 104: 34$ & $1,822: 86: 7^{* *}$ & $3,264: 190: 41$ \\
\hline Encephalopathy, none:grade I-II:grade III-IV & $1,578: 2: 0$ & $1,857: 53: 5^{* *}$ & $3,435: 55: 5$ \\
\hline ICG-R15, $\%^{\mathrm{a}}$ & $21.0(13.0-33.6)$ & $16.0(10.6-23.0)^{* *}$ & $17.4(11.3-28.1)$ \\
\hline Liver damage, A:B:C & $823: 658: 99$ & $1,137: 735: 43^{* *}$ & $1,960: 1,393: 142$ \\
\hline Child-Pugh, A:B:C & $1,266: 282: 32$ & 1,568:329:18* & $2,834: 611: 50$ \\
\hline ALBI grade, $1: 2: 3$ & $670: 834: 76$ & $792: 1,086: 37^{* *}$ & $1,462: 1,920: 113$ \\
\hline Tumor size, $\mathrm{cm}^{\mathrm{a}}$ & $2.3(1.6-3.6)$ & $2.4(1.6-3.5)$ & $2.3(1.6-3.5)$ \\
\hline Tumor number, single:multiple & $956: 624$ & $1,328: 587^{* *}$ & $2,284: 1211$ \\
\hline TNM stage of LCSGJ, I:II:III:IV & 408:728:345:99 & $574: 914: 371: 56^{* *}$ & $982: 1,642: 716: 155$ \\
\hline AFP, ng/mL, <100: $\geq 100: N D$ & $1,181: 393: 6$ & $1,055: 277: 583^{* *}$ & $2,236: 670: 589$ \\
\hline AFP-L3, $\%,<10: \geq 10: N D$ & $1,057: 378: 145$ & $859: 271: 785$ & $1,916: 649: 930$ \\
\hline $\mathrm{DCP}, \mathrm{mAU} / \mathrm{mL},<100: \geq 100: \mathrm{ND}$ & $917: 638: 25$ & $856: 436: 623^{* *}$ & $1,773: 1,074: 648$ \\
\hline Treatment, resection:RFA:PEI:TACE:other & $400: 833: 40: 265: 42$ & 874:819:11:197:14** & $1,274: 1,652: 51: 462: 56$ \\
\hline
\end{tabular}

${ }^{*} p<0.05,{ }^{* *} p<0.01$, comparison between Ehime and validation groups. IQR, interquartile range; HCV, hepatitis C virus; HBV, hepatitis B virus; nonBnonC, negative for both HBV and HCV; AST, aspartate amino transferase; ALT, alanine transferase; ICG-R15, indocyanine green retention rate after $15 \mathrm{~min}$; ALBI grade, albumin-bilirubin grade; TNM stage, tumor node metastasis stage; LCSGJ, Liver Cancer Study Group of Japan; ND, no data; AFP, $\alpha$-fetoprotein; AFP-L3, lens culinaris agglutinin A-reactive fraction of AFP; DCP, des- $\gamma$-carboxy prothrombin; RFA, radiofrequency ablation; PEI, percutaneous ethanol injection; TACE, transcatheter arterial chemoembolization. ${ }^{a}$ Median (IQR).

Table 3. Comparison of patient distribution between liver damage, Child-Pugh, and ALBI grade

\begin{tabular}{|c|c|c|c|c|c|c|}
\hline & \multicolumn{3}{|c|}{ Ehime group $(n=1,580)$} & \multicolumn{3}{|c|}{ Validation group $(n=1,915)$} \\
\hline & LD A & LD B & LD C & LD A & LD B & LD C \\
\hline ALBI-1 & 557 & 113 & 0 & 719 & 73 & 0 \\
\hline ALBI-2 & 266 & 527 & 41 & 417 & 645 & 24 \\
\hline \multirow[t]{2}{*}{ ALBI-3 } & 0 & 18 & 58 & 1 & 17 & 19 \\
\hline & LD A & LD B & LD C & LD A & LD B & LD C \\
\hline CP A & 822 & 440 & 4 & 1,131 & 435 & 2 \\
\hline CP B & 1 & 206 & 75 & 6 & 293 & 30 \\
\hline \multirow[t]{2}{*}{$\mathrm{CP} \mathrm{C}$} & 0 & 12 & 20 & 0 & 7 & 11 \\
\hline & CP A & CP B & $\mathrm{CP} \mathrm{C}$ & CP A & СР B & $\mathrm{CP} C$ \\
\hline ALBI-1 & 664 & 6 & 0 & 785 & 7 & 0 \\
\hline ALBI-2 & 602 & 223 & 9 & 783 & 297 & 6 \\
\hline ALBI-3 & 0 & 53 & 23 & 0 & 25 & 12 \\
\hline
\end{tabular}

LD, liver damage; CP, Child-Pugh; ALBI, albumin-bilirubin grade. 


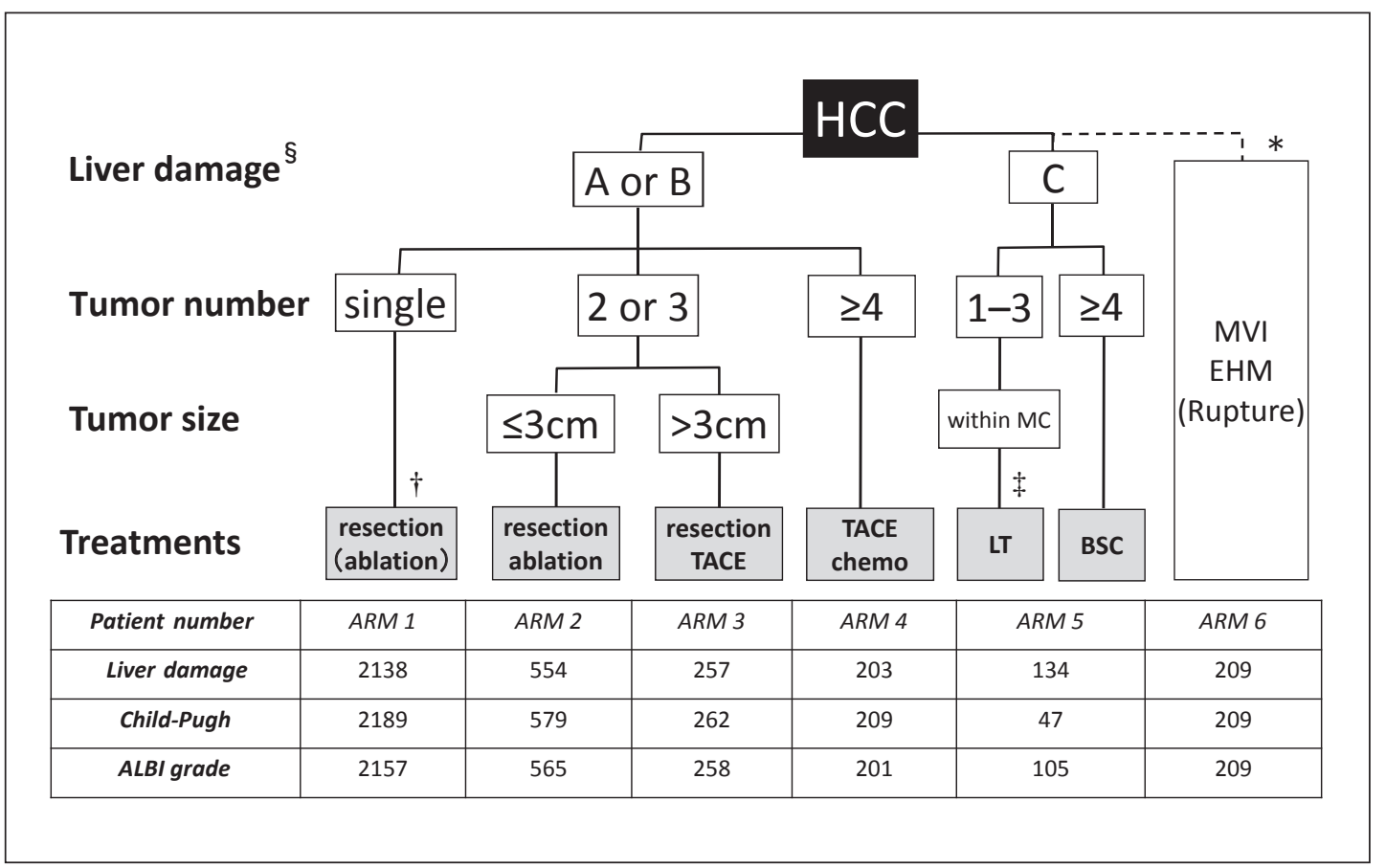

Fig. 1. HCC treatment algorithm of the evidence-based Japan Society of Hepatology (JSH) guideline and distribution of patients with 3 hepatic function assessment tools. ${ }^{\S}$ Child-Pugh classification may also be used when nonsurgical treatment is considered. ${ }^{\dagger}$ Can be selected for tumors with a diameter $\leq 3 \mathrm{~cm}$. ${ }^{\ddagger}$ Patients aged $\leq 65$ years. * In some cases, liver resection, chemotherapy, and embolization therapy may be selected for patients with Child-Pugh class A along with vascular invasion, while chemotherapy is recommended for patients with Child-Pugh class A disease with extrahepatic metastasis. TACE, transcatheter arterial chemoembolization; MC, Milan criteria (single lesion $\leq 5 \mathrm{~cm}$ or $2-3$ lesions each $\leq 3 \mathrm{~cm}$ ); LT, liver transplantation; BSC, best supportive care; MVI, major vessels invasion; EHM, extrahepatic metastasis; ALBI grade, albuminbilirubin grade.

$2,189,579,262,209$, and 47, respectively, and using ALBI grade were 2,157, 565, 258, 201, and 105, respectively (Fig. 1).

Prognosis for each grade according to each hepatic function assessment method was also examined (Fig. 2a-c). The value obtained with the AIC for prognosis by ALBI grade was lower as compared to the liver damage and Child-Pugh values $(22,291.8,22,379.6$, and 22,392.1, respectively). Similarly, that for prognosis according to ALBI-T score was lower than those according to modified JIS and JIS scores $(21,989.4,22,076.0$, and 22,075.1, respectively) (Fig. 2d-f).

The distribution of levels of ICG-R15 in each assessment is shown in Figure 3. Those of ICG-R15 $<10 \%, 10$ to $<20 \%, 20$ to $<30 \%$, and $\geq 30 \%$ for liver damage class A and B were similar to those for ALBI grade 1 and 2 (Fig. 3). ALBI score showed a good relationship with ICG-R15 ( $r=0.616, p<0.001$ ) (Fig. 4a). The cutoff values for ALBI score obtained by calculating ROC and AUC values for prediction of ICG-R15 $(<10 \%)$ were -2.623 and 0.798 , respectively, while those for ICG-R15 $(<20 \%)$ were -2.470 and 0.791 , respectively, and for ICG-R15 $(<30 \%)$ were -2.222 and 0.843 , respectively (Fig. 4 b-d). 


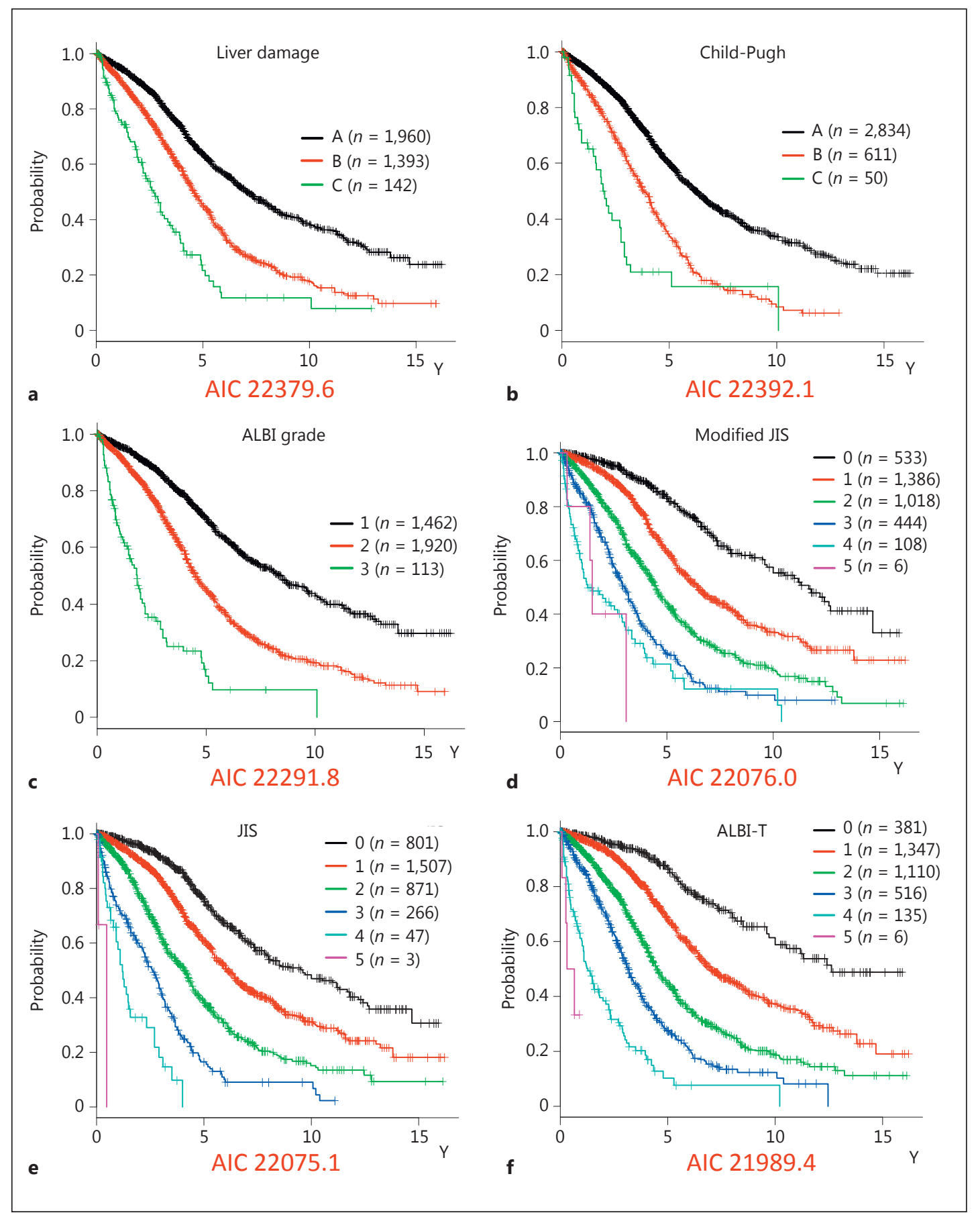

Fig. 2. a Overall survival rates of patients according to liver damage classification (Akaike information criterion, AIC: 22,379.6). b Overall survival rates of patients according to the Child-Pugh classification (AIC: 22,392.1). c Overall survival rates of patients according to albumin-bilirubin (ALBI) grade (AIC: 22,291.8). d Overall survival rates of patients according to modified Japan Integrated scoring system (JIS) score (AIC: 22,076.0). e Overall survival rates of patients according to JIS score (AIC: 22,075.1). f Overall survival rates of patients according to ALBI-tumor node metastasis (ALBI-T) score (AIC: 21,989.4). 


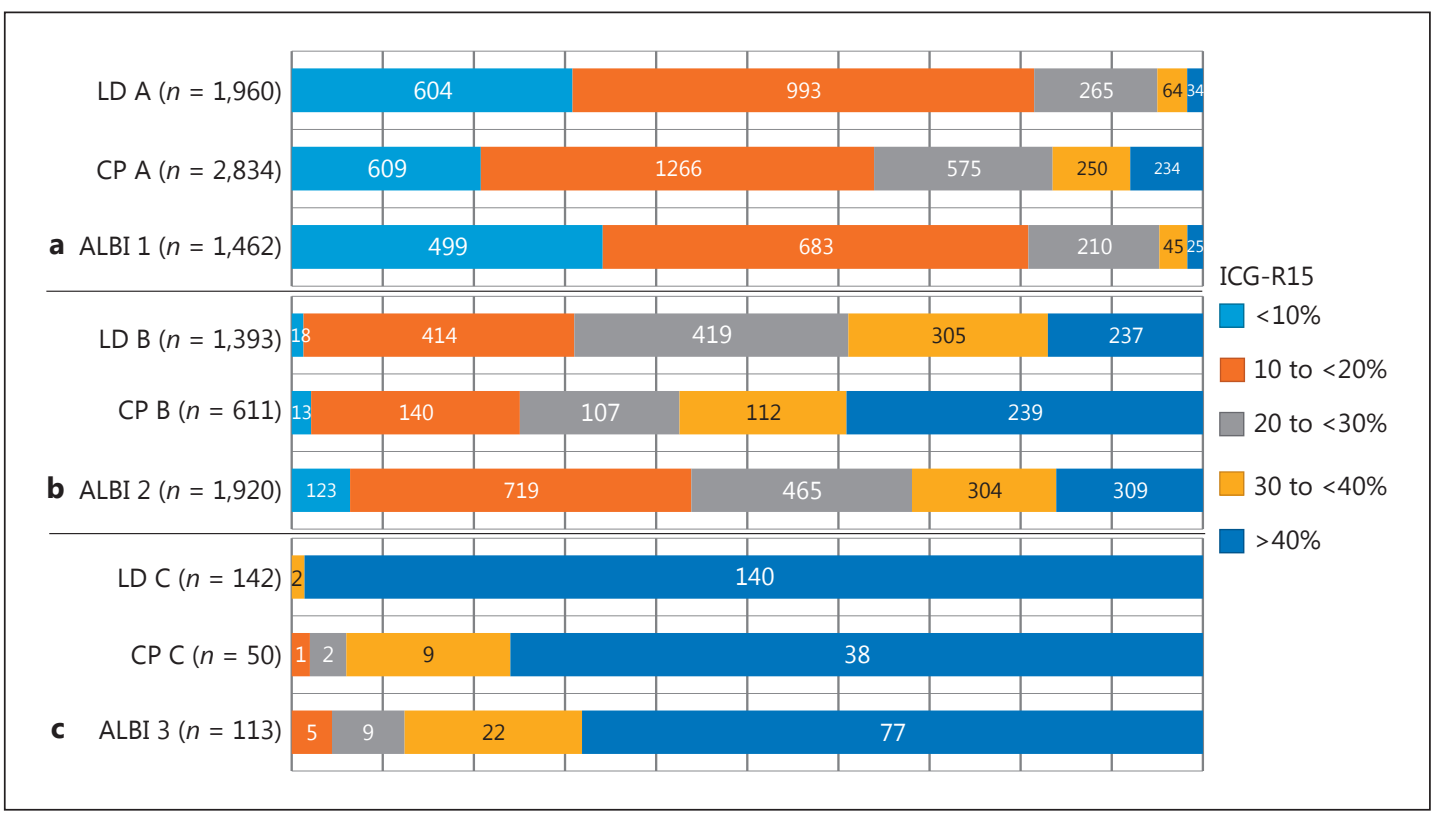

Fig. 3. Distribution of level of indocyanine green retention rate at 15 min (ICG-R15). a Comparison between liver damage (LD) class A, Child-Pugh (CP) class A, and albumin-bilirubin (ALBI) grade 1. b Comparison between LD class B, CP class B, and ALBI grade 2. c Comparison between LD class C, CP class C, and ALBI grade 3.

\section{Discussion}

Therapy for HCC in Japanese patients is generally performed according to the HCC treatment algorithm described in the "guidelines for liver cancer treatment based on scientific evidence" using the liver damage classification in the evidence-based JSH guideline [2,3]. The liver damage classification, which requires ICG test results, has been reported to be more effective for evaluation of hepatic function than the Child-Pugh classification [26, 27]. On the other hand, the Child-Pugh classification may also be used in the evidence-based JSH guideline for consideration of nonsurgical treatment [3]. In fact, the ICG test was performed only in $46.2 \%$ of the present patients with HCC in all institutions. In addition, there are issues related to bolus injection of ICG, needed for an ICG test, and allergy to ICG, and constitutional ICG excretory defect [28] and the possibility of underestimation of hepatic function for patients who have a progressed portal-venous shunt [29] have been reported. Moreover, there might also be problems when liver damage and Child-Pugh, which has been reported to be inferior to liver damage [8], are used together in the evidence-based JSH guideline. The newly proposed ALBI grade [9] was shown to provide a better estimation of hepatic function than the ChildPugh classification [13], and can be calculated with only albumin and total bilirubin data. Indeed, no need for imaging assessment of ascites, encephalopathy, subjective evaluation, or ICG-R15 is thought to be an advantage of ALBI grade as compared with the other classifications. In the present analysis, when ALBI grade was used for assessment of hepatic function in the Japanese HCC treatment algorithm, the distribution of patients in each therapeutic option arm was similar as compared to the liver damage classification.

No comparisons between the liver damage classification and ALBI grade have previously been made. In the present study, the Ehime and validation groups showed a similar tendency for predicting liver damage class A between ALBI grade and the Child-Pugh classification, and 


\section{cearcer}

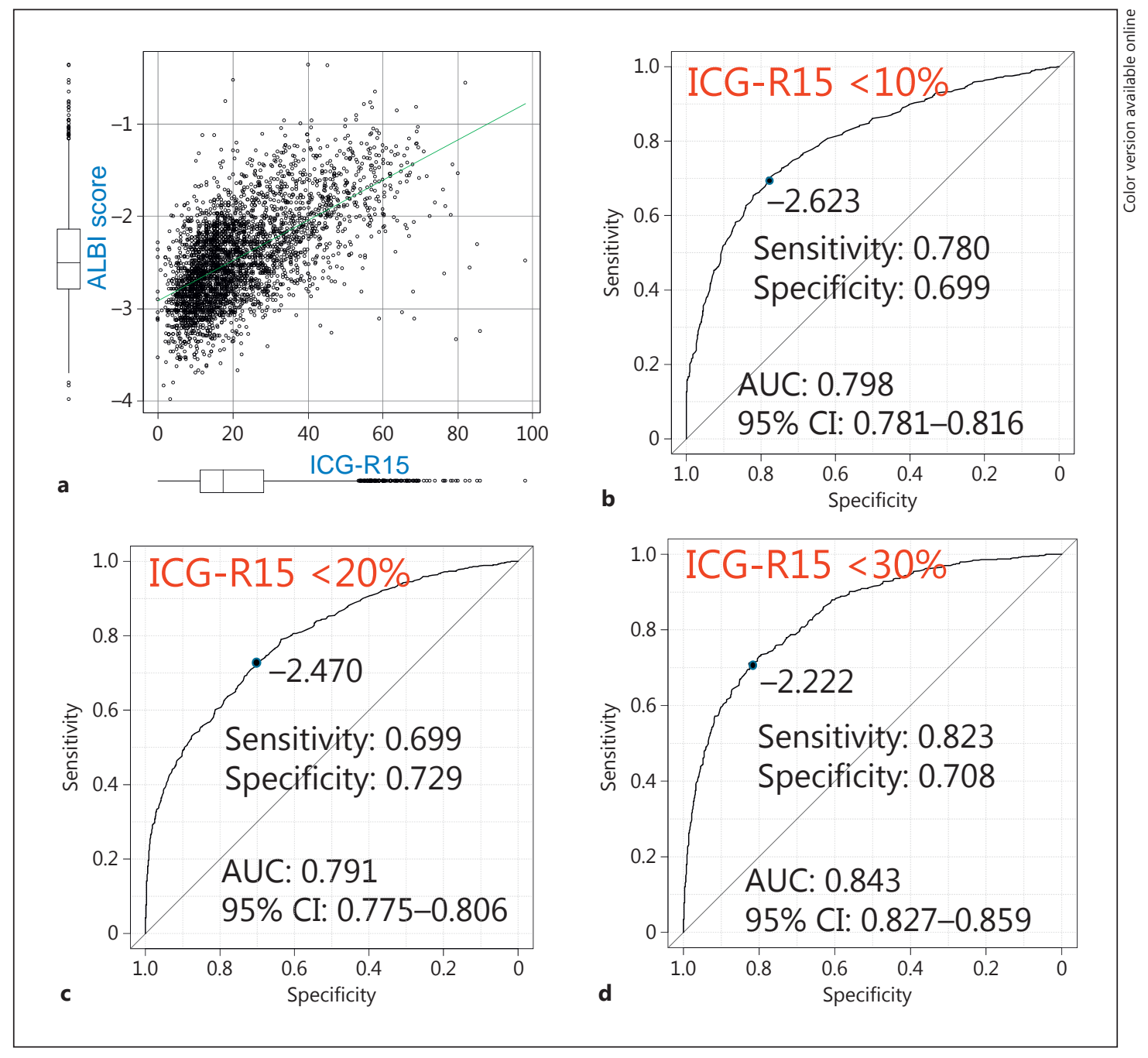

Fig. 4. a Relationship between albumin-bilirubin (ALBI) score and indocyanine green retention rate at 15 $\min (I C G-R 15)(r=0.616, p<0.001)$. $\mathbf{b}$ The cutoff value of ALBI score for predicting ICG-R15 $(<10 \%)$ by calculating receiver operating characteristic (ROC) was -2.623 (sensitivity: 0.780, specificity 0.699, AUC: 0.798). c The cutoff value of ALBI score for predicting ICG-R15 (<20\%) by ROC was -2.470 (sensitivity: 0.699, specificity 0.729, AUC: 0.791). $\mathbf{d}$ The cutoff value of ALBI score for predicting ICG-R15 (<30\%) by ROC was -2.222 (sensitivity: 0.823 , specificity 0.708 , AUC: 0.843 ).

the positive likelihood ratio of ALBI grade 1 for liver damage class A was good. However, as compared to the Child-Pugh classification, the frequency of patients categorized as requiring transplantation or supportive care due to decompensated classification (arm 5) was increased in the order of ALBI grade and liver damage. Child-Pugh classification had a tendency to overestimate, while liver damage had a tendency to underestimate hepatic function. In a comparison of AIC for prognosis assessment, ALBI grade and ALBI-T score were better than the others. These results suggest that ALBI grade has a superior ability for assessment of hepatic function in patients with HCC.

Surgical resection plays a very important role for improving the prognosis of HCC patients, especially in those with a low number of small-sized tumors (e.g., Milan criteria [30]). Bennett and Blumgart [31] reported that ICG-R15 was unnecessary for deciding on the indication for resection or selection of surgery. However, the ICG test has been described in many reports 
as an important examination in patients treated with surgical resection. Makuuchi and colleagues noted the efficacy of ICG test results for decision making in regard to resectable area and proposed the Makuuchi criteria [6,32], while Torzilli et al. [33] reported satisfactory results following hepatic resection, with a 30 -day postoperative mortality of $0 \%$ when the criteria were considered for selecting patients to undergo surgery. Ariizumi et al. [34] also reported that the rates of postoperative liver failure and mortality were higher in patients with ICG-R15 (10\% or higher) compared to others who underwent a right hepatectomy or trisectionectomy for HCC ( 23 vs. $2 \%, p=0.002$, and 11 vs. $0 \%, p=0.016$, respectively).

In addition to the ICG test, development of intraoperative ultrasonically guided subsegmentectomy techniques [35, 36] has made surgical resection safer [37]. In a follow-up survey conducted in Japan $(n=54,145)$, hospital mortality after a resection procedure was reported to be $2.6 \%$ [38]. When evaluation for hepatic function was done using ALBI grade, most patients with ALBI grade 1 showed an ICG-R15 value lower than 30\%. The Makuuchi criteria define resectable segments according to ICG-R15 level $(<10 \%=>2$ segments, 10 to $<20 \%=$ 1 segment, 20 to $<30 \%$ = subsegment, 30 to $<40 \%=$ partial, $\geq 40 \%=$ enucleation) [6, 32]. In the present study, the distribution of ICG-R15 levels in ALBI grade 1 was similar to that in liver damage class A. Based on this result, we consider that the ICG test can be used if necessary, depending on the area scheduled for hepatectomy (e.g., subsegmental, segmental, lobectomy) in patients graded as ALBI-1, and when hepatectomy is considered for patients graded as ALBI-2. Thus, ALBI grade might help to solve difficulties associated with performing the ICG test in HCC patients and the inferiority of assessment with the Child-Pugh classification for hepatic function.

The present study has some limitations, including its retrospective nature. Further investigations with greater numbers of cases are needed to confirm our findings. The majority of the present cohort was considered for resection or RFA, and additional patients with liver damage class $C$ should be analyzed. Of course, each parameter is only one of many indicators available for estimation of hepatic function, and a group of patients cannot be clearly divided into different therapeutic strategies using only a single parameter. Nevertheless, our findings indicate that ALBI grade is a simple but informative clinical indicator for planning treatment against HCC and is considered as adequate for use in the HCC algorithm.

\section{Disclosure Statement}

None of the authors has financial conflicts of interest to disclose concerning this study.

\section{References}

1 Liver Cancer Study Group of Japan: The General Rules for the Clinical and Pathological Study of Primary Liver Cancer, ed 6. Tokyo, Kanehara \& Co, 2015, pp 26-30.

2 Kokudo N, Hasegawa K, Akahane M, Igaki H, Izumi N, Ichida T, Uemoto S, Kaneko S, Kawasaki S, Ku Y, Kudo M, Kubo S, Takayama T, Tateishi R, Fukuda T, Matsui O, Matsuyama Y, Murakami T, Arii S, Okazaki M, Makuuchi M: Evidence-based Clinical Practice Guidelines for Hepatocellular Carcinoma: The Japan Society of Hepatology 2013 update (3rd JSH-HCC Guidelines). Hepatol Res 2015;45:123-127.

3 The Japan Society of Hepatology: Clinical Practice Guidelines for Hepatocellular Carcinoma 2013. http:// www.jsh.or.jp/English/examination_en.

4 Greco E, Nanji S, Bromberg IL, Shah S, Wei AC, Moulton CA, Greig PD, Gallinger S, Cleary SP: Predictors of periopertative morbidity and liver dysfunction after hepatic resection in patients with chronic liver disease. HPB (Oxford) 2011;13:559-565.

5 Lau H, Man K, Fan ST, Yu WC, Lo CM, Wong J: Evaluation of preoperative hepatic function in patients with hepatocellular carcinoma undergoing hepatectomy. Br J Surg 1997;84:1255-1259.

6 Makuuchi M, Kosuge T, Takayama T, Yamazaki S, Kakazu T, Miyagawa S, Kawasaki S: Surgery for small liver cancers. Semin Surg Oncol 1993;9:298-304. 
7 Pugh RN, Murray-Lyon IM, Dawson JL, Pietroni MC, Williams R: Transection of the oesophagus for bleeding oesophageal varices. Br J Surg 1973;60:646-649.

8 Omagari K, Ohba K, Kadokawa Y, Hazama H, Masuda J, Kinoshita H, Matsuo I, Ohnita K, Mizuta Y, Hayashida K, Kohno S: Comparison of the grade evaluated by "Liver damage" of Liver Cancer Study Group of Japan and Child-Pugh classification in patients with hepatocellular carcinoma. Hepatol Res 2006;34:266-272.

9 Johnson PJ, Berhane S, Kagebayashi C, Satomura S, Teng M, Reeves HL, O’Beirne J, Fox R, Skowronska A, Palmer D, Yeo W, Mo F, Lai P, Iñarrairaegui M, Chan SL, Sangro B, Miksad R, Tada T, Kumada T, Toyoda H: Assessment of liver function in patients with hepatocellular carcinoma: a new evidence-based approach-the ALBI grade. J Clin Oncol 2015;33:550-558.

10 Wang YY, Zhong JH, Su ZY, Huang JF, Lu SD, Xiang BD, Ma L, Qi LN, Ou BN, Li LQ: Albumin-bilirubin versus Child-Pugh score as a predictor of outcome after liver resection for hepatocellular carcinoma. Br J Surg 2016; 103:725-734.

11 Toyoda H, Lai PB, O’Beirne J, Chong CC, Berhane S, Reeves H, Manas D, Fox RP, Yeo W, Mo F, Chan AW, Tada T, Iñarrairaegui M, Vogel A, Schweitzer N, Chan SL, Sangro B, Kumada T, Johnson PJ: Long-term impact of liver function on curative therapy for hepatocellular carcinoma: application of the ALBI grade. Br J Cancer 2016; 114:744-750.

12 Hiraoka A, Kumada T, Nouso K, Tsuji K, Itobayashi E, Hirooka M, Kariyama K, Ishikawa T, Tada T, Toyoda H, Kawasaki H, Hiasa Y, Michitaka K: Proposed new sub-grouping for intermediate-stage hepatocellular carcinoma using albumin-bilirubin grade. Oncology 2016;91:153-161.

13 Hiraoka A, Kumada T, Michitaka K, Toyoda H, Tada T, Ueki H, Kaneto M, Aibiki T, Okudaira T, Kawakami T, Kawamura T, Yamago H, Suga Y, Miyamoto Y, Tomida H, Azemoto N, Mori K, Miyata H, Ninomiya T, Kawasaki $\mathrm{H}$ : Usefulness of albumin-bilirubin grade for evaluation of prognosis of 2584 Japanese patients with hepatocellular carcinoma. J Gastroenterol Hepatol 2016;31:1031-1036.

14 Edeline J, Blanc JF, Johnson P, Campillo-Gimenez B, Ross P, Ma YT, King J, Hubner RA, Sumpter K, Darby S, Evans J, Iwuji C, Swinson D, Collins P, Patel K, Muazzam I, Palmer DH, Meyer T: A multicenter comparison between Child Pugh and ALBI scores in patients treated with sorafenib for hepatocellular carcinoma. Liver Int 2016;36:1821-1828.

15 Chan AW, Kumada T, Toyoda H, Tada T, Chong CC, Mo FK, Yeo W, Johnson PJ, Lai PB, Chan AT, To KF, Chan SL: Integration of albumin-bilirubin (ALBI) score into Barcelona Clinic Liver Cancer (BCLC) system for hepatocellular carcinoma. J Gastroenterol Hepatol 2016;31:1300-1306.

16 Chan AW, Chong CC, Mo FK, Wong J, Yeo W, Johnson PJ, Yu S, Lai PB, Chan AT, To KF, Chan SL: Incorporating Albumin-Bilirubin grade into the Cancer of the Liver Italian Program system for hepatocellular carcinoma. J Gastroenterol Hepatol, Epub ahead of print.

17 Kudo M, Chung H, Haji S, Osaki Y, Oka H, Seki T, Kasugai H, Sasaki Y, Matsunaga T: Validation of a new prognostic staging system for hepatocellular carcinoma: the JIS score compared with the CLIP score. Hepatology 2004;40:1396-1405.

18 Kudo M, Chung H, Osaki Y: Prognostic staging system for hepatocellular carcinoma (CLIP score): its value and limitations, and a proposal for a new staging system, the Japan Integrated Staging Score (JIS score). J Gastroenterol 2003;38:207-215.

19 Ikai I, Takayasu K, Omata M, Okita K, Nakanuma Y, Matsuyama Y, Makuuchi M, Kojiro M, Ichida T, Arii S, Yamaoka Y; Liver Cancer Study Group of Japan: A modified Japan Integrated Stage score for prognostic assessment in patients with hepatocellular carcinoma. J Gastroenterol 2006;41:884-892.

20 Bruix J, Sherman M: Management of hepatocellular carcinoma. Hepatology 2005;42:1208-1236.

21 Hiraoka A, Hiasa Y, Onji M, Michitaka K: New contrast enhanced ultrasonography agent: impact of Sonazoid on radiofrequency ablation. J Gastroenterol Hepatol 2011;26:616-618.

22 Kanda Y: Investigation of the freely available easy-to-use software "EZR" for medical statistics. Bone Marrow Transplant 2013;48:452-458.

23 R Development Core Team RFfSC: R: A Language and Environment for Statistical Computing. Vienna, The R Foundation for Statistical Computing, 2005.

24 Hiraoka A, Michitaka K, Horiike N, Hidaka S, Uehara T, Ichikawa S, Hasebe A, Miyamoto Y, Ninomiya T, Sogabe I, Ishimaru Y, Kawasaki H, Koizumi Y, Hirooka M, Yamashita Y, Abe M, Hiasa Y, Matsuura B, Onji M: Radiofrequency ablation therapy for hepatocellular carcinoma in elderly patients. J Gastroenterol Hepatol 2010;25: 403-407.

25 Hiraoka A, Horiike N, Yamashita Y, Koizumi Y, Doi K, Yamamoto Y, Hasebe A, Ichikawa S, Yano M, Miyamoto Y, Ninomiya T, Otomi Y, Kokame M, Iwamura T, Ishimaru Y, Sogabe I, Kashihara K, Nishiura S, Ootani H, Takamura $\mathrm{K}$, Kawasaki H: Efficacy of radiofrequency ablation therapy compared to surgical resection in 164 patients in Japan with single hepatocellular carcinoma smaller than $3 \mathrm{~cm}$, along with report of complications. Hepatogastroenterology 2008;55:2171-2174.

26 Watanabe Y, Kumon K: Assessment by pulse dye-densitometry indocyanine green (ICG) clearance test of hepatic function of patients before cardiac surgery: its value as a predictor of serious postoperative liver dysfunction. J Cardiothorac Vasc Anesth 1999;13:299-303.

27 Erdogan D, Heijnen BH, Bennink RJ, Kok M, Dinant S, Straatsburg IH, Gouma DJ, van Gulik TM: Preoperative assessment of liver function: a comparison of $99 \mathrm{mTc}-M e b r o f e n i n$ scintigraphy with indocyanine green clearance test. Liver Int 2004;24:117-123. 
28 Taketazu F, Sanada I, Ngamatsu N, Mukai R, Suetomo Y, Toyoda N, Takada M, Hida K, Kubota K, Maezawa M, Takaku F: A case of hereditary elliptocytosis associated with constitutional indocyanine green excretory defect. Jpn J Med 1984;23:139-143.

29 Hiraoka A, Kurose K, Hamada M, Azemoto N, Tokumoto Y, Hirooka M, Hasebe A, Kumagi T, Hirata M, Michitaka K, Minami H, Murakami M, Isobe Y, Horiike N, Onji M: Hepatic encephalopathy due to intrahepatic portosystemic venous shunt successfully treated by interventional radiology. Intern Med 2005;44:212-216.

30 Mazzaferro V, Regalia E, Doci R, Andreola S, Pulvirenti A, Bozzetti F, Montalto F, Ammatuna M, Morabito A, Gennari L: Liver transplantation for the treatment of small hepatocellular carcinomas in patients with cirrhosis. N Engl J Med 1996;334:693-699.

31 Bennett JJ, Blumgart LH: Assessment of hepatic reserve prior to hepatic resection. J Hepatobiliary Pancreat Surg 2005;12:10-15.

32 Imamura H, Seyama Y, Kokudo N, Maema A, Sugawara Y, Sano K, Takayama T, Makuuchi M: One thousand fifty-six hepatectomies without mortality in 8 years. Arch Surg 2003;138:1198-1206; discussion 1206.

33 Torzilli G, Makuuchi M, Inoue K, Takayama T, Sakamoto Y, Sugawara Y, Kubota K, Zucchi A: No-mortality liver resection for hepatocellular carcinoma in cirrhotic and noncirrhotic patients: is there a way? A prospective analysis of our approach. Arch Surg 1999;134:984-992.

34 Ariizumi S, Yamamoto M, Takasaki K: Right hepatectomy for hepatocellular carcinoma in patients with an indocyanine green retention rate at 15 minutes of 10\% or higher. Dig Surg 2009;26:135-142.

35 Makuuchi M, Hasegawa H, Yamazaki S: Ultrasonically guided subsegmentectomy. Surg Gynecol Obstet 1985; 161:346-350.

36 Makuuchi M, Hasegawa H, Yamazaki S: Intraoperative ultrasonic examination for hepatectomy. Ultrasound Med Biol 1983;suppl 2:493-497.

37 Kudo M, Izumi N, Ichida T, Ku Y, Kokudo N, Sakamoto M, Takayama T, Nakashima O, Matsui O, Matsuyama Y: Report of the 19th follow-up survey of primary liver cancer in Japan. Hepatol Res 2016;46:372-390.

38 Ikai I, Arii S, Okazaki M, Okita K, Omata M, Kojiro M, Takayasu K, Nakanuma Y, Makuuchi M, Matsuyama Y, Monden M, Kudo M: Report of the 17th Nationwide Follow-up Survey of Primary Liver Cancer in Japan. Hepatol Res 2007;37:676-691. 